\title{
Near wake of emergent vegetation patches in shallow flow
}

\author{
Sina Wunder ${ }^{1, *}$, Michele Trevisson ${ }^{1, * *}$, Christoph Heckele ${ }^{1}$, Lö̈c Chagot $^{2}$, Brendan Murphy ${ }^{3}$, \\ Stuart McLelland ${ }^{3}$, Frédéric Moulin ${ }^{2}$, and Olivier Eiff ${ }^{1, * * *}$ \\ ${ }^{1}$ Karlsruher Institut für Technologie, Institut für Hydromechanik, Kaiserstr.12, D-76131 Karlsruhe - \\ Germany \\ ${ }^{2}$ Institut de Mécanique des Fluides de Toulouse (IMFT), UMR 5502, 2 Allée du Professeur Camille \\ Soula, F-31400 Toulouse - France \\ ${ }^{3}$ University of Hull, School of Environmental Sciences, HULL, HU6 7RX - Great Britain
}

\begin{abstract}
Vegetation patches are particularly difficult to quantify in terms of flow resistance due to their complex geometry and topological behaviour under hydrodynamic loading. They not only influence the water level and mean velocities due to the drag they exert, but they also affect the turbulence and hence all transfer processes such as the sediment transport dynamics in the surrounding area. Existing studies dealing with the interaction of flow and vegetation concern mostly measurements of the drag of single plants followed by analyses of the flow through and above homogeneous canopies. However, studies of the flow around single patches are uncommon and are mostly restricted to arrays of cylindrical elements. For leafy plants there is very limited information and understanding of how the flow evolves through and around the plants. This work aims at filling these gaps via complementary physical lab-scale and numerical experiments of the flow through and around an artificial vegetation patch. The experimental work focuses on PIV measurements in the wake of the patches whereas the method of large-eddy simulation is employed to provide additional insights of the flow inside the patch. Here we focus on results based on the PIV measurements.
\end{abstract}

\section{Introduction}

The prediction of flow velocities and water levels during flood events is nowadays still inaccurate due to difficulty in accounting for emergent and submerged obstacles resisting the flow. Particularly vegetation patches are difficult to quantify in terms of flow resistance and of their effect on the flow field (such as turbulence and transfer processes) due to their complex geometry and topological behavior under hydrodynamic loading. Thus, the flow resistance and interactions depend on the allometric characteristics of the vegetation patch itself. These are generally described by frontal density, solid volume fraction, leaf area index (LAI), shape and flexibility, with major uncertainties both in their flow-dependent

\footnotetext{
*e-mail: sina.wunder@kit.edu

**e-mail: michele.trevisson@kit.edu

***e-mail: olivier.eiff@kit.edu
} 
determination and parameterization, necessary for implementation in larger-scale numerical models ([1], [5], [9] and [12]).

Existing investigations on the vegetative drag were mostly performed by direct force measurements using the classical one-parameter drag force equation for solid bodies to parameterize the effects of the allometric plant characteristics by modifying the velocity dependence with additional parameters ([2], [1], [19]). However, as recent studies on the flow through emergent single vegetation patches ([15] and [14]) have shown, the use of such parameterization of the drag force equation is questionable when the plant is significantly penetrated by the flow. To avoid these parametrizations, difficult to justify, studies have started to investigate the flow itself around emergent patches constructed by arrayed cylinders, to better characterize the flow pattern and turbulence structures in the wake zone ([18], [17]). In these studies the flow was characterized by instantaneous and local velocity measurements. Yet, most of the local flow investigations did not focus on the question of the drag parametrization but on the morphodynamic processes within and downstream of the emergent patches ([7], [10] and [11]). PIV-measurements around patches have so far only been performed in the wake zone of a single and submerged artificial fractal tree ([3] and [4]). Numerical (RNG and LES) investigations of the flow field within and around a patch have also been performed and have so far considered emergent arrays of solid cylinders ([6], [13] and [14]) or artificial plants ([5], [16] and [20]).

In summary, for leafy plants there is still very limited information and understanding of how the flow through them and the resulting drag evolves. This paper proposes start filling these gaps by performing PIV-measurements in transverse planes just up- and downstream of an artificial vegetation patch, meeting the allometric characteristics of a real leafy plant. The study reveals new information about the flow structure and the turbulence field.

\section{Experimental set-up}

In the present experimental study, the mean and turbulent flow around a single artificial patch with the same solid volume fraction and leaf area index (LAI) of a real plant were investigated in a shallow configuration to analyse the effect of the penetrating flow on the flow pattern just around the patch. The experiments were performed in the recirculating Total Environment Simulator of Hull University, Great Britain, a glass-walled flume $6 \mathrm{~m}$ wide and $10 \mathrm{~m}$ long. The large width of the flume enabled to minimize side wall effects.
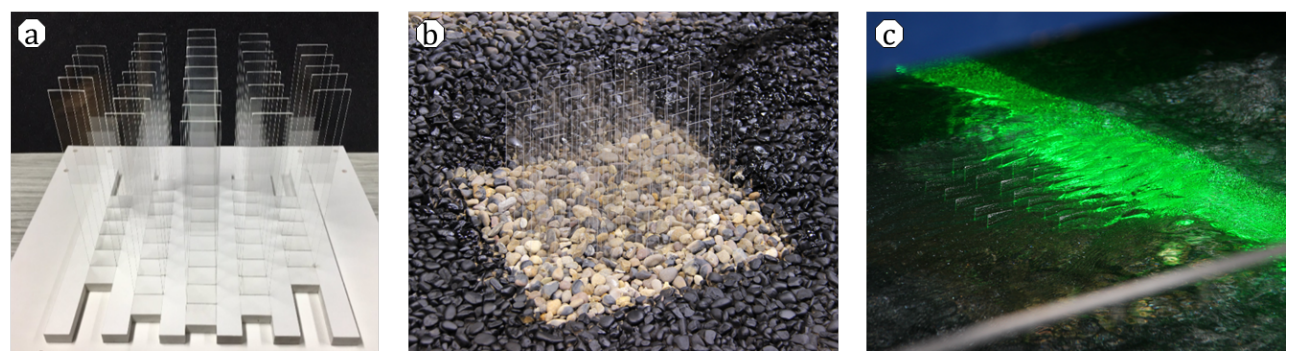

Figure 1. Design of the artificial patch (a), meeting the allometric characteristics of a natural willow plant, installed in the flume (b) and during experiments (c). 
A patch, consisting of a geometrically ordered structure, designed with 35 vertical glass plates, $1 \mathrm{~mm}$ thin, $4 \mathrm{~cm}$ long and $20 \mathrm{~cm}$ high, arranged in a staggered configuration (cf. figure 1), was installed in the center of the flume (reduced to $3 \mathrm{~m}$ width). The bed of the flume was covered by gravel with mean diameter of $d_{g}=2 \mathrm{~cm}$ (cf. figure 1b) and a slope of $I_{0}=0.0015$. To cover the allometric parameters of a real shrubby plant, the design of the geometry (number of plates per base area) met the natural values of solid volume fraction, i.e. porosity $(\phi=0.98)$, and the leaf area index $(L A I=4.0)$ of a real shrubby willow.

The flow field around the patch was characterized by stereoscopic 2D-3C PIV measurements in two transverse vertical planes: upstream of the patch in a distance of $x_{1}=3.4 \mathrm{~cm}$ to the first row of glass sheets and downstream of the patch again in a distance of $x_{2}=3.4 \mathrm{~cm}$ to the last row of glass sheets. It was assumed that the flow field behaves symmetrically with respect to the centerline in flow direction. Measurements were performed for relative submergence of plant-height to water-depth $h_{p} / h=1$, a bulk flow velocity of $U_{b}=0.42 \mathrm{~m} / \mathrm{s}$ and a flow depth of $h=0.2 \mathrm{~m}$. The Reynolds-number of the channel flow $R_{f}$ and the leaf Reynolds-number $R e_{l}$ were equal to $\operatorname{Re}_{f}=U_{b} \cdot h / v=6.5 \cdot 10^{4}$ and $\operatorname{Re}_{p}=U_{b} \cdot l / v=1.3 \cdot 10^{4}$, respectively, where $l$ is the length of the leafes in streamwise direction. Thus, turbulent flow conditions prevailed.

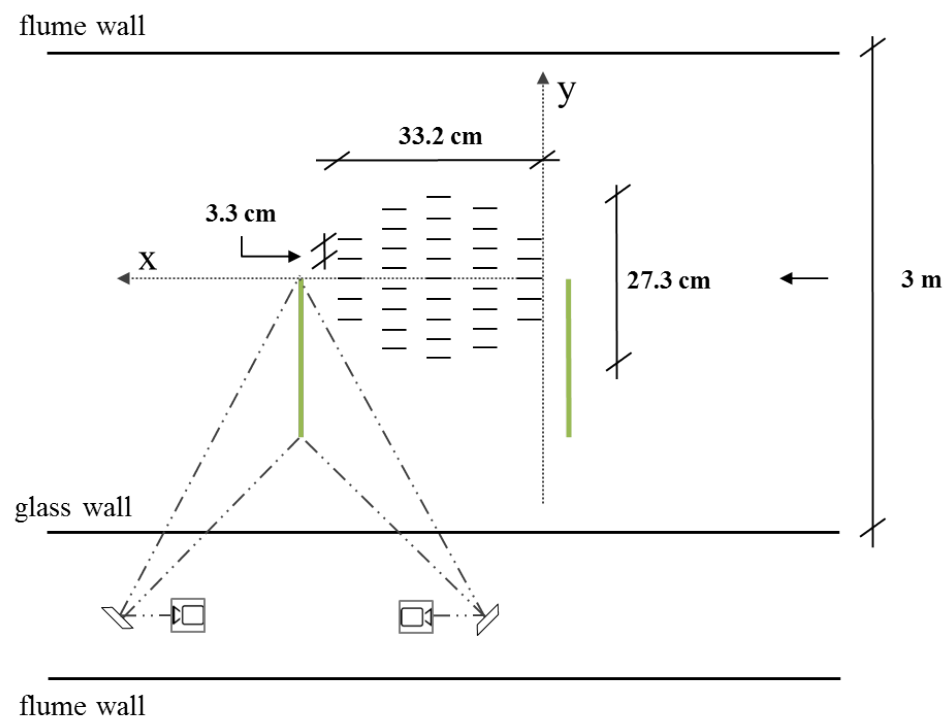

Figure 2. Set-up of the stereoscopic 2D-3C PIV measurements around the left side patch in longitudinal (left figure) and transversal (right figure) direction (Not to scale).

The instrumentation used constisted of a 2 x $120 \mathrm{~mJ}$ pulsed laser of Dantec Dynamics producing a $10 \mathrm{~mm}$ thick laser sheet synchronized with two motorised cameras with a 65 $\mathrm{mm}$ lens. The cameras were positioned in a $1.5 \mathrm{~m}$ long underwater-torpedo, that recorded the images via movable mirrors with resolution of 2320 pixels x 1726 pixels. The seeding used was PLASCOAT TALISMAN 20 with a mean density of $\rho=0.99 \mathrm{~g} / \mathrm{cm}^{3}$ and a particle size of $d_{s} \approx 180 \mu \mathrm{m}$. To converge statistically, 5000 image pairs with a sampling rate of $4 \mathrm{~Hz}$ were collected for each camera. 


\section{Results and discussion}

Owing to the stereoscopic measurement technique, the three components of the velocity field in each measurement plane of both investigated cross sections were obtained. Figure 3 shows the time-averaged cross sectional velocity distribution in the flow direction $\bar{u}(y, z)$ including the vector field of the vertical and horizontal time-averaged components $\bar{v}$ and $\bar{w}$ of the upstream (cf. figure 3a) and downstream (cf. figure 3b) cross section. The transverse positions of the glass sheet rows are illustrated by dashed lines: the black lines show the nearest row of glass sheets with respect to the measured cross section, the dark grey lines show the first recessed row of glass sheets and the white lines show the second recessed row with largest distance. Additionally the horizontal profile of the depth-averaged velocity profiles $\bar{u}_{d}$ normalized by the global bulk velocity $U_{b}$ in both measured cross sections is shown (cf. figure 3c).
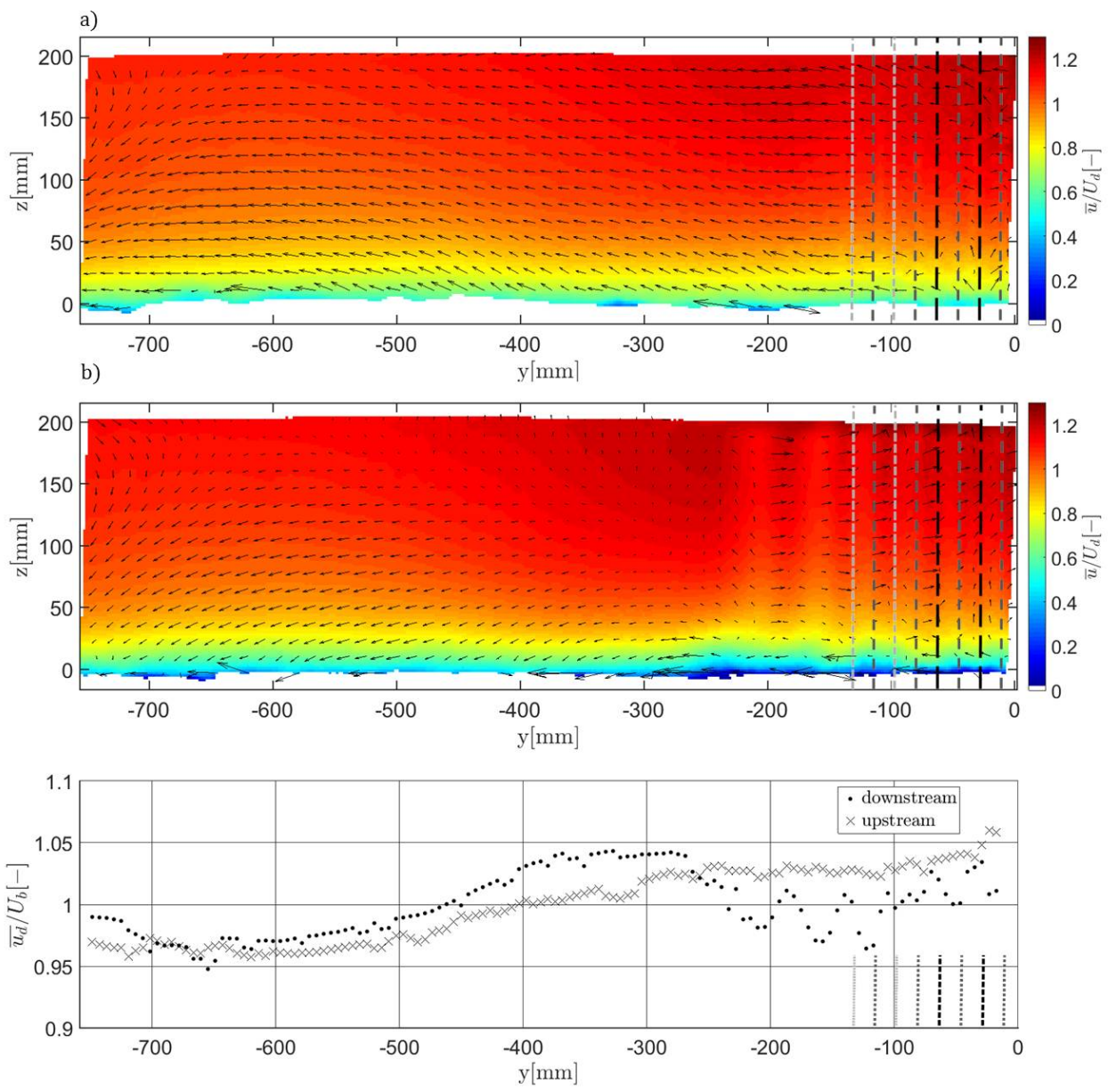

Figure 3. Contourplot of the time averaged flow velocities in mean flow direction including the vector field of lateal and vertical time averaged components upstream (a) and downstream (b) of the patch and a horizontal depth averaged velocity profile of both cross sections (c). Every third data point is plotted. 
It can be seen in figure 3a that upstream of the patch, an acceleration of the mean flow along the periphery of the patch develops. The patch affects in this manner the flow over a width three times its own. Despite this acceleration around the patch, the streamwise velocity is also accelerated just before penetrating into the patch. The intensity of this effect increases towards the centerline, which was also found in [8]. It can also be observed that the lateral and vertical velocity components upstream of the patch are inhomogenously distributed, particularly near the bed and near the water surface (cf. figure $3 \mathrm{a}$ ).

In the near wake the influence of the staggered glass sheets on the flow pattern is even more significant as the wake consists of vertical fields of reduced flow velocity, alternated with regions of higher flow velocity (cf. figure $3 \mathrm{~b} / \mathrm{c}$ ). This is clearly a signature of the individual glass plates rather than a global wake. The columnar variations suggest a pattern of prolonged separated boundary layers of the single glass sheets. The acceleration of the peripheric part still affects the flow over the same distance from the patch as upstream, but the width of the columnar patch wake exceeds the patch width by a factor of 1.5 . Thus, the individual wakes do not follow the flow direction but are deviated towards the periphery. This deviation is quite strong over a short distance. In contrast to the acceleration near the patch, the periphery beyond the wakes is characterized by lower flow velocities, which can also be observed in the upstream plane. By comparing the depth-averaged velocity profiles (cf. figure $3 \mathrm{c}$ ) it can be seen, that the flow has not changed far from the patch, accelerates in near periphery and decelerates by passing through the patch, as expected.

Figure 4 shows the horizontal profiles of the $\overline{u^{\prime} v^{\prime}}$ Reynolds-stresses at the downstream cross section at three heights, near the bed at $z=50 \mathrm{~mm}$ (dotted line), within the water body at $z=125 \mathrm{~mm}$ (dashed line) and near the water surface at $z=200 \mathrm{~mm}$ (solid line). The positions of the glass sheet rows again are illustrated by dashed lines as in figure 3. Additionally, the curve of the depth-averaged flow velocity of this cross-section is included in the figure.

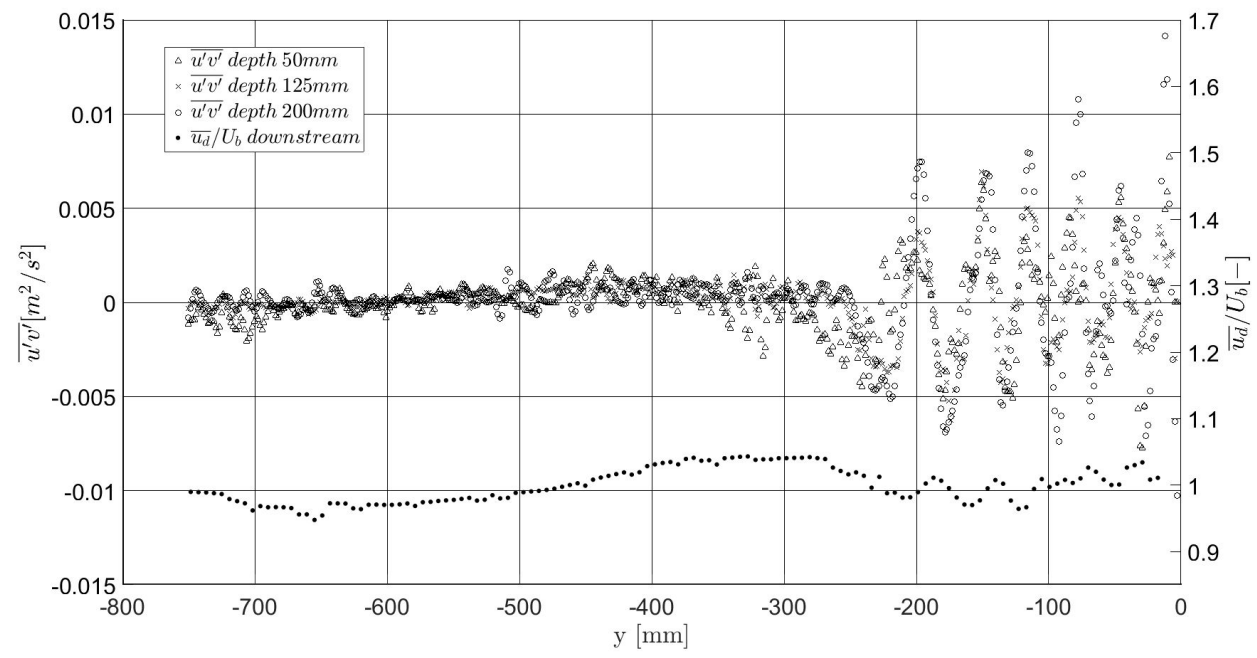

Figure 4. Horizontal profiles of the $\overline{u^{\prime} v^{\prime}}$ Reynolds-stresses downstream of the patch within the water body $(z=125 \mathrm{~mm})$, near the water surface $(z=200 \mathrm{~mm})$ and above the gravel bed $z=50 \mathrm{~mm})$, including the depth-averaged flow velocity from figure $3 \mathrm{c}$. 
The Reynolds-stresses are marked by positive and negative oscillations towards the centerline and towards the periphery they are essentially constant. The oscillations (seven) are clearly the signature of the columnar individual wakes since all three levels are essentially aligned. Again, these wakes have shifted towards the periphery and are not aligned with the rows of glass plates. The lateral deviation also leads to a widening of the wakes, increasing towards the periphery. The signs of the peak Reynolds stresses align well with the corresponding maximum lateral gradients of the depth-averaged mean longitudinal velocity, a signature of the individual wakes.

Interestingly, there the larger scale Reynolds-stress variation is very weak, in correspondance with the weak larger-than-wake scale mean velocity gradients. Clearly, there is no patch-toouterflow mixing layer. Regarding the wakes again, it can also be observed that, first, the Reynolds stresses increase from the bed towards the free surface, suggesting that the bed boundary-layer weakens the plate boundary-layers and / or the wakes. Second, the Reynolds stress peaks increase towards the centerline, in accordance with the fact that the number of glass plates in a row increases towards the centerline. This suggests a superposition of successive individual wakes.

\section{Conclusion}

In this experimental investigation by 2D-3C stereocopic PIV measurements the turbulent flow in lateral planes just upstream and downstream of a patch was studied. The patch consisted of vertical thin glass sheets in a staggered configuration and was installed into a gravel-bed covered flume and studied under emergent conditions. The geometry of the patch was defined by meeting the allometric characteristics of a natural plant, such as leaf area index (LAI) and solid volume fraction. The stereoscopic yielded the three-componentvelocity fields. The staggered geometry of the thin glass sheets lead to a development of local boundary layers, resulting in alternating vertical wakes downstream of the patch. These wakes are clearly marked by Reynolds-stresses due to lateral shear in the wakes. The wakes are also shifted outwards towards the periphery without generating a significant larger scale shear towards the outer flow. Nevertheless, the impact of the patch on the mean flow is about three times its width. In further analysis, the behavior of the wake with increasing distance to the patch compared to the flow pattern of a real plant will be studied.

\section{Acknowledgements}

The model tests were conducted by H+-HULL-01 User Group in the Total Environment Simulator, University of Hull, UK, under Contract Number 654110 of the European Community. The financial contribution from the EC is appreciated.

\section{References}

[1] Aberle, J., Järvelä, J.: Flow resistance of emergent rigid and flexible floodplain vegetation, J. Hydr. Res. 51(1), 33-45 (2013)

[2] Armanini, A., Righetti, M., Grisenti, P.: Direct measurement of vegetation resistance in prototype scale, J. Hydr. Res. 43 (5), 481-487 (2005)

[3] Bai, K., Meneveau, C., Katz, J.: Near-wake turbulent flow structure and mixing length downstream of a fractal tree, Boundary-Layer Meteorol. (143), 285-308 (2012) 
[4] Bai, K., Meneveau, C., Katz, J.: Experimental study of spectral energy fluxes in turbulence generated by a fractal, treelike object, Phys. Fluids 25, 110810 (2013)

[5] Boothroyd, R. J., Hardy, R. J., Warburton, J., Marjoribanks, T. I.: The importance of accurately representing submerged vegetation morphology in the numerical prediction of complex river flow. Earth Surf. Process. and Landf.2015

[6] Chang, W.-Y., Contantinescouu, G., Tsai, W. F.: On the flow and coherent structures generated by a circular array of rigid emerged cylinders placed in an open channel with flat and deformed bed. J. Fluid Mech. 831, 1-40 (2017)

[7] Chen, Z., Ortiz, A., Zong, L., Nepf, H.: The wake structure behind a porous obstruction and its implications for deposition near a finite patch of emergent vegetation. Water Res. Res. 48 (9), 2012

[8] Dupuis, V., Moulin, F., Cazin, S., Moise, M., Elyakime, P., Barron, J., Eiff, O.: Shallow flow over a bed with lateral change of roughness. RiverFlow, 2018, submitted.

[9] Jalonen, J., Järvelä, J., Aberle, J.: Leaf area index as a vegetation density measure for hydraulic analyses. J. Hydr. Eng. 139(5), 461-469 (2013)

[10] Kim,H. S., Kimura, I., Shimizu, Y.: Bed morphological changes around a finite patch of vegetation, Earth Surf. Process. Landforms 40, 375-388 (2015)

[11] Liu, C., Nepf, H.: Sediment deposition within and around a finite patch of model vegetation over a range of channel velocity, Water Resour. Res. 52, (2016)

[12] Marjoribanks, T. I., Hardy, R. J., Lane, S. N., Parsons, D. R.: The hydraulic description of vegetated river channels: the weaknesses of existing formulations and emerging alternatives. Wiley Interdisciplinary Reviews: Water 11/2014 1(6), (2014)

[13] Marjoribanks, T. I., Hardy, R. J., Lane, S. N., Parsons, D. R.: High-resolution numerical modelling of flow-vegetation interactions. J. Hydr. Res. 52(6), 775-793 (2014)

[14] Nicolle, A., Eames, I.: Numerical study of flow through and around a circular array of cylinders, J. Fluid Mech. 679, 1-31 (2011)

[15] Rominger, J. T., Nepf, H. M.: Flow adjustment and interior low associated with a rectangular porous obstruction, J. Fluid Mech. 680, 636-659 (2011)

[16] Stoesser, T.; Kim, S. J.; Diplas, P.: Turbulent flow through idealized emergent vegetation,. J. Hydr. Eng. 136 (12), 1003-1017 (2010)

[17] Tanino, Y.; Nepf, H. M.: Laboratory investigation of mean drag in a random array of rigid, emergent cylinders, J. Hydr. Eng. 134 (1), 34-41 (2008)

[18] Thomas, R. E, McLelland, S. J.: The impact of macroalgae on mean and turbulent flow fields, J. Hydrodyn. 27(3), 427-435 (2015)

[19] Wunder, S., Lehmann, B., Nestmann, F.: Determination of the drag coefficients of emergent and just submerged willows, J. River Basin Management 9 (3-4), 231-236 (2011)

[20] Zong, L., Nepf, H.: Vortex development behind a finite porous obstruction in a channel, J. Fluid Mech. 691, 368-391 (2011) 